\title{
polymers
}

ISSN 2073-4360

www.mdpi.com/journal/polymers

Article

\section{Polymerization in Liquid Crystal Medium: Preparation of Polythiophene Derivatives Bearing a Bulky Pyrimidine Substituent}

\author{
Satoshi Ohkawa, Reina Ohta, Kohsuke Kawabata and Hiromasa Goto * \\ Institute of Materials Science, Graduate School of Pure and Applied Sciences, University of Tsukuba, \\ Tsukuba, Ibaraki, 305-8573, Japan
}

* Author to whom correspondence should be addressed; E-Mail: gotoh@ ims.tsukuba.ac.jp; Tel.: +81-29-853-5128; Fax: +81-29-853-4490.

Received: 4 August 2010; in revised form: 25 September 2010 / Accepted: 29 September 2010 / Published: 11 October 2010

\begin{abstract}
We carried out polycondensation of monomers bearing a bulky pyrimidine substituent in a liquid crystal solvent. The resultant polymers formed nematic liquid crystals. The polymers prepared in liquid crystals had higher coplanarity than the polymers prepared in toluene. This can be due to the fact that the ordered medium of the liquid crystal produces an aggregated structure with well-developed $\pi$-stacking between the main chains. The present results demonstrated that polymerization of bulky monomers is possible in liquid crystal solvents.
\end{abstract}

Keywords: liquid crystal; $\pi$-conjugated polymer; $\pi$ stacking

\section{Introduction}

Liquid crystals (LCs) have fluidity and crystal-like order [1-12]. Previous studies reported syntheses of polythiophenes bearing alkyl groups in LCs as anisotropic media [13-15]. The resultant polymers possessed the structural order derived from the LC media employed for the polymerization. The polymerization process was influenced by the ordered structures of LCs and resulted in formation of LC order for the resultant polymers. Such studies have reported successful polymerization in the LC 
media from monomers that displayed good affinity towards LCs. Polymerization in LC media may require a certain combination of molecular forms of the LCs and monomers.

In the present study, following from the successful polymerization of the aromatic main chain type polymer bearing flexible alkyl group, we conducted polycondensation of monomers bearing a bulky substituent in LCs to explore the possibility of polymerization in LC. Thermotropic behavior of the polymers thus obtained is investigated with differential scanning calorimetry (DSC) and visual observation of the optical textures is carried out with polarizing optical microscopy (POM). Furthermore, optical properties such as optical absorption and photoluminescence are discussed based on consideration of aggregation derived from inter-chain interaction of the polymers. The results of this study may open the possibility of various polymerization reactions in liquid crystals as reaction media.

\section{Experimental Section}

\subsection{Materials and methods}

All syntheses except the Williamson etherification reaction in the present study were conducted under argon atmosphere. 4-(5-Nonyl-pyrimidine-2-yl)-phenol (compound 1) was provided by Midori Chemicals and used for side chains of polymers. 2,5-Bis-trimethyl-stannyl thiophene and 5,5'-bis(trimethylstannyl)-2,2'-bithiophene were synthesized by a previously reported method [16]. ${ }^{1}$ H-NMR spectra were measured with JNM-ECS 400 (JEOL) and EX-270 (JEOL) NMR spectrometers. $\mathrm{CDCl}_{3}$ was employed for the NMR solvent. Chemical shifts are represented in parts per million downfield from tetramethylsilane (TMS) as an internal standard. ${ }^{1} \mathrm{H}_{-}{ }^{13} \mathrm{C}$ hetero-nuclear multiple quantum coherence (HMQC) NMR were measured with an AVANCE-500 Spectrometer (Bruker). Infrared absorption spectra were measured with an FT/IR-300 spectrometer (JASCO). UV-vis absorption spectra of the polymers in chloroform were measured with a V-630 UV-vis optical absorption spectrometer (JASCO). Photoluminescence spectra of the polymers in chloroform were measured with an F-4500 fluorescence spectrophotometer (HITACHI). Optical textures of the LC and the polymers were observed with an ECLIPSE LV100 polarizing optical microscope (Nikon) equipped with a TH-600PM (Linkam) thermo control stage. The samples were sandwiched between two thin glass slides for the POM observations. Differential scanning calorimetry (DSC) analysis was conducted with a DSC6200 (SEIKO Instruments).

\subsection{Synthesis of cholesteric liquid crystal medium}

A cholesteric liquid crystal medium, $(R)$-LC, was synthesized according to the literature procedure [13]. Scheme 1 shows the chemical structure of the $(R)$-LC. The $(R)$-LC has a mesogen which consists of three-ring and flexible alkyl chains at both ends of the molecule, which allows the formation of the LC phase under appropriate conditions.

Liquid crystals having three-ring aromatic cores tend to be in the liquid crystal state at high temperatures $\left(>100{ }^{\circ} \mathrm{C}\right)$. In fact, the $(R)$-LC exhibits a liquid crystal phase at $78-137{ }^{\circ} \mathrm{C}$. Therefore, $(R)$-LC can be employed as the reaction medium for the Migita-Kosugi-Stille type polycondensation reactions, which require high reaction temperatures $\left(>100{ }^{\circ} \mathrm{C}\right)$. 
Scheme 1. Chemical structure of (R)-LC.

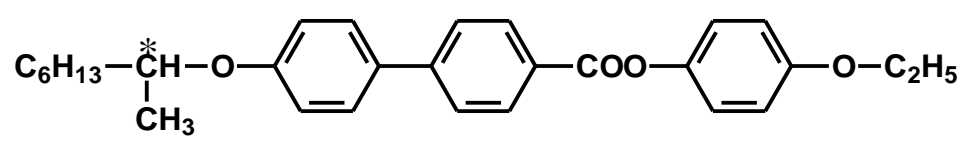

\subsection{Synthesis of monomer}

A synthetic route for 2,5-dibromo-benzoic acid 6-[4-(5-nonyl-pyrimidine-2-yl)-phenoxy]-hexyl ester (mono-1) is shown in Scheme 2. First, 6-chloro-1-hexanol was coupled with compound 1 via Williamson etherification reaction. Compound 1 (1.26 mmol), 6-chloro-1-hexanol (1.32 mmol), 18-crown-6-ether $(0.063 \mathrm{mmol})$, and $\mathrm{K}_{2} \mathrm{CO}_{3}(1.26 \mathrm{mmol})$ were dissolved in $N, N$-dimethylformamide (DMF, $0.8 \mathrm{~mL}$ ), and the mixture was refluxed at $120{ }^{\circ} \mathrm{C}$ for $20 \mathrm{~h}$. After the reaction, the solution was extracted with dichloromethane and washed with water. Recrystallization from ethanol followed by vacuum drying afforded compound 2 as a white solid $(0.60 \mathrm{mmol}$, Yield $(\mathrm{Y})=48 \%)$. The successful synthesis of compound $\mathbf{2}$ was confirmed by ${ }^{1} \mathrm{H}-\mathrm{NMR}$ spectroscopy.

Scheme 2. Synthetic route for mono-1.

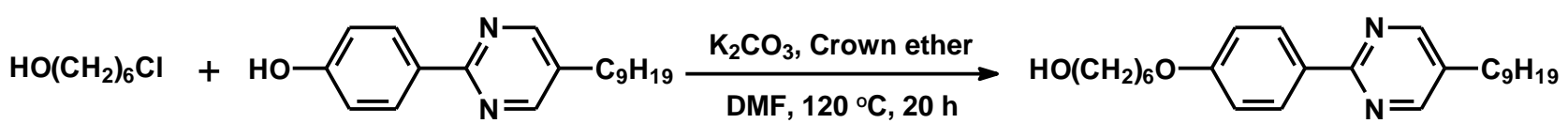

Compound 1

Compound 2

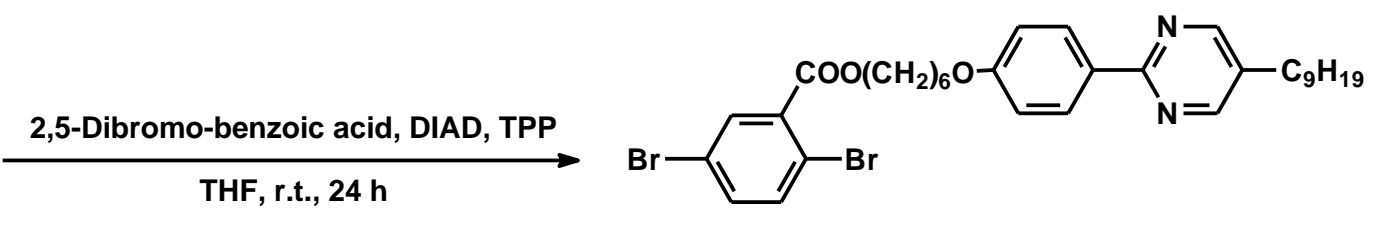

Mono-1

Next, compound 2 was coupled with 2,5-dibromobenzoic acid via Mitsunobu reaction [17]. 2,5-Dibromobenzoic acid $(0.6 \mathrm{mmol})$, compound $2(0.6 \mathrm{mmol})$ and triphenylphosphine (TPP) $(0.72 \mathrm{mmol})$ were dissolved in tetrahydrofuran (THF, $3.5 \mathrm{~mL})$. Diisopropyl azodicarboxylate (DIAD) ( $40 \%$ in toluene) $(0.66 \mathrm{mmol})$ was slowly added to this solution in an ice bath at $0{ }^{\circ} \mathrm{C}$. The reaction mixture was warmed up to room temperature and stirred for $24 \mathrm{~h}$. After completion of the reaction, the solution was extracted with dichloromethane and washed with water. The solution was then evaporated and crude solid was purified by silica gel column chromatography using chloroform/methanol $(\mathrm{v} / \mathrm{v}=20 / 1)$ as an eluent. Recrystallization from ethanol followed by vacuum drying afforded the target compound (mono- 1$)$ as a white solid $(0.34 \mathrm{mmol}, \mathrm{Y}=56 \%)$. The structure of mono-1 was confirmed by ${ }^{1} \mathrm{H}-\mathrm{NMR}$ spectroscopy and ${ }^{1} \mathrm{H}-{ }^{13} \mathrm{C}$ heteronuclear multiple quantum coherence (HMQC) NMR spectroscopy (Chart 1). Carbon atoms and protons in aromatic ring exhibit correlated signals at low magnetic region (a-1). Signals due to carbon atoms and protons adjacent to the ether oxygen atoms are observable as correlation signals d-4 and f-6, respectively. Protons and carbons in pyrimidine ring appear at very low magnetic field region as correlation signals i-9. 
Chart 1. ${ }^{1} \mathrm{H}-{ }^{13} \mathrm{C}$ hetero-nuclear multiple quantum coherence (HMQC) NMR of mono- 1.

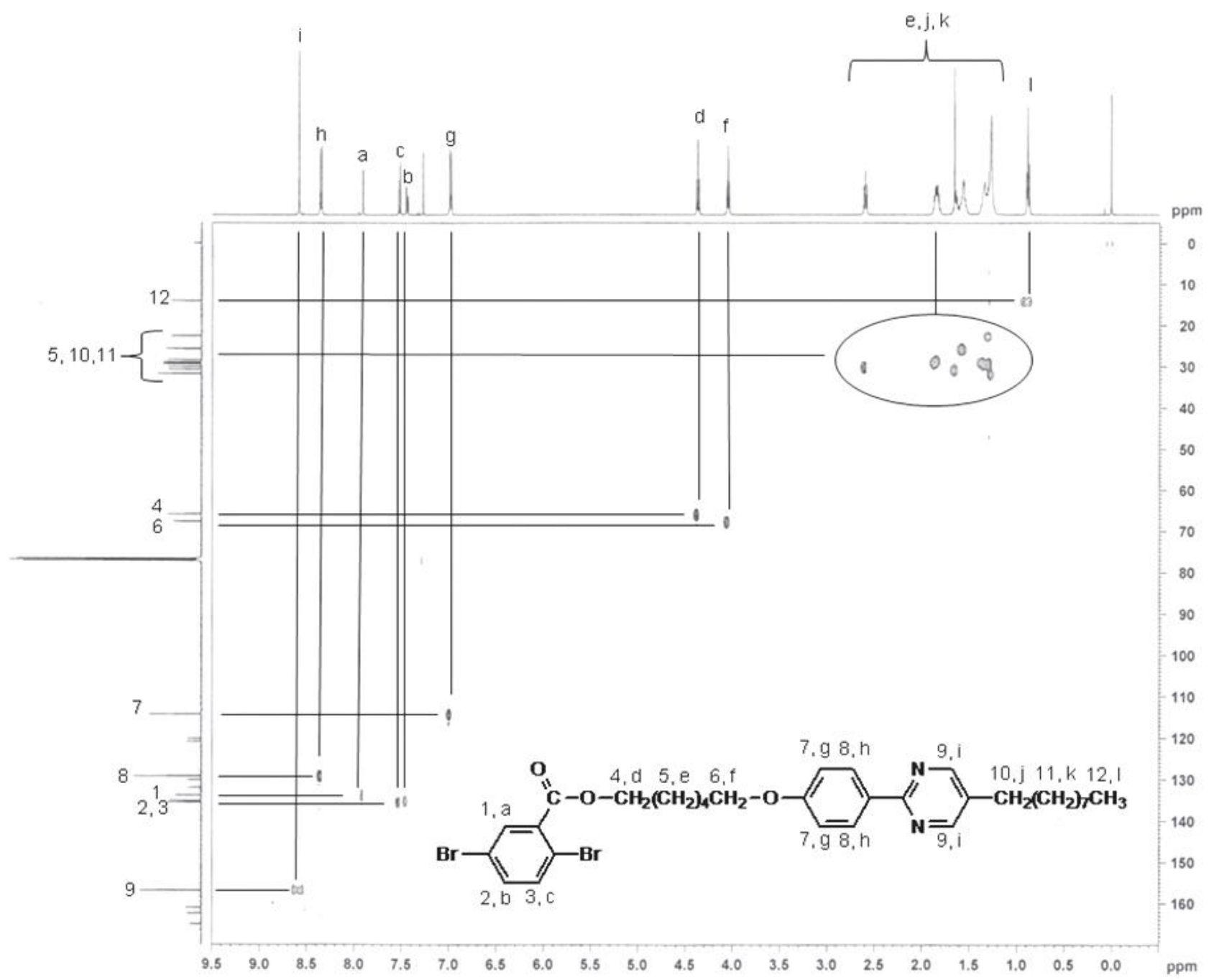

${ }^{1} \mathrm{H}-\mathrm{NMR}$ for compound $2\left(270 \mathrm{MHz}, \mathrm{CDCl}_{3}, \delta\right.$ from TMS): $\delta 8.57(\mathrm{~s}, 2 \mathrm{H}), 8.34(\mathrm{~d}, 2 \mathrm{H}, J=8.75 \mathrm{~Hz})$, $6.97(\mathrm{~d}, 2 \mathrm{H}, J=8.75 \mathrm{~Hz}), 4.03(\mathrm{t}, 2 \mathrm{H}, J=6.48 \mathrm{~Hz}), 3.67(\mathrm{q}, 2 \mathrm{H}, J=5.97 \mathrm{~Hz}), 2.60(\mathrm{t}, 2 \mathrm{H}$, $J=7.67 \mathrm{~Hz}), 1.81(\mathrm{t}, 2 \mathrm{H}, J=7.43 \mathrm{~Hz}), 1.65-1.20(\mathrm{~m}, 20 \mathrm{H}), 0.88(\mathrm{t}, 3 \mathrm{H}, J=6.48 \mathrm{~Hz})$.

${ }^{1} \mathrm{H}-\mathrm{NMR}$ for mono-1 (400 MHz, $\mathrm{CDCl}_{3}, \delta$ from TMS): $\delta 8.57(\mathrm{~s}, 2 \mathrm{H}), 8.34(\mathrm{dd}, 2 \mathrm{H}, J=9.14 \mathrm{~Hz}$, $2.32 \mathrm{~Hz}), 7.90(\mathrm{~d}, 1 \mathrm{H}, J=2.28 \mathrm{~Hz}), 7.51(\mathrm{~d}, 1 \mathrm{H}, J=8.39 \mathrm{~Hz}), 7.43(\mathrm{dd}, 1 \mathrm{H}, J=8.23 \mathrm{~Hz}, 2.28 \mathrm{~Hz})$, $6.97(\mathrm{dd}, 2 \mathrm{H}, J=9.14 \mathrm{~Hz}, 2.28 \mathrm{~Hz}), 4.36(\mathrm{t}, 2 \mathrm{H}, J=6.60 \mathrm{~Hz}), 4.04(\mathrm{t}, 2 \mathrm{H}, J=6.60 \mathrm{~Hz}), 2.60(\mathrm{t}, 2 \mathrm{H}$, $J=7.59 \mathrm{~Hz}), 1.88-1.79(\mathrm{~m}, 4 \mathrm{H}), 1.63-1.55(\mathrm{~m}, 6 \mathrm{H}), 1.33-1.26(\mathrm{~m}, 12 \mathrm{H}), 0.88(\mathrm{t}, 3 \mathrm{H}, J=6.79 \mathrm{~Hz})$.

\subsection{Polymerization}

Scheme 3 shows Migita-Kosugi-Stille type polycondensation reaction between mono-1 and 2,5-bis-trimethyl-stannyl thiophene or 5,5'-bis(trimethylstannyl)-2,2'-bithiophene using the $\operatorname{Pd}(0)$ catalyst in toluene or $(R)$-LC. Each procedure is described below. 
Scheme 3. Syntheses of the polymers.

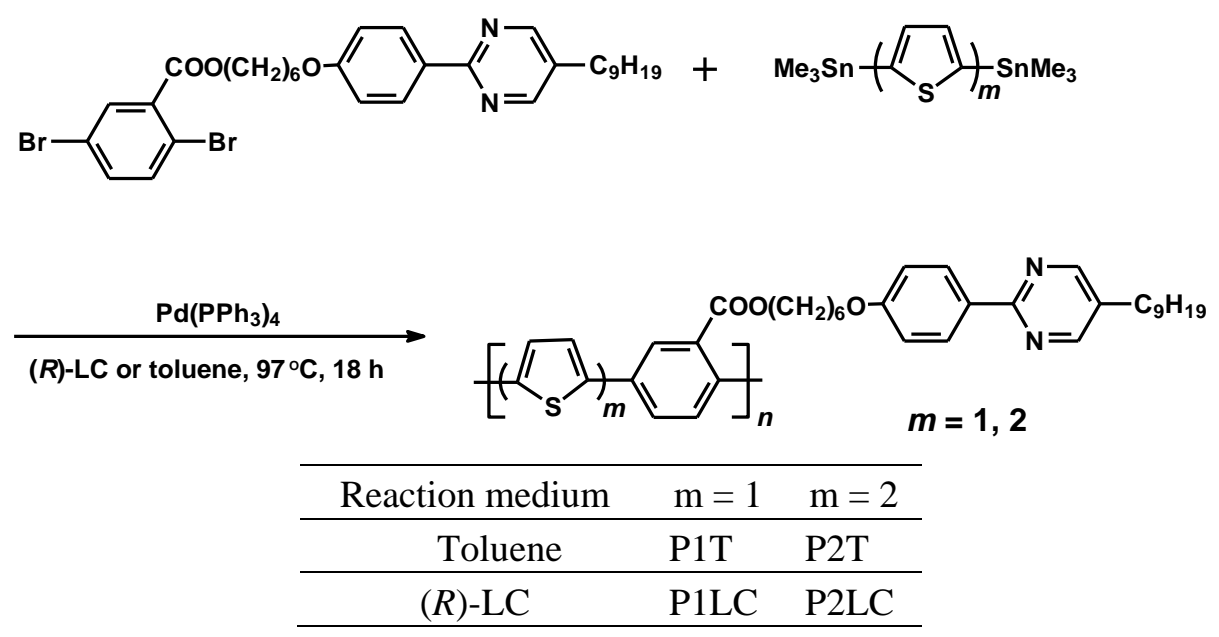

Table 1. Polymerization results.

\begin{tabular}{ccccc}
\hline Polymer & $M_{\mathrm{n}}{ }^{[\mathrm{a}]}$ & $M_{\mathrm{w}}{ }^{[\mathrm{a}]}$ & $M_{\mathrm{w}} / M_{\mathrm{n}}{ }^{[\mathrm{a}]}$ & Yield [\%] \\
\hline P1T & 4700 & 6900 & 1.5 & 90 \\
\hline P2T & 3800 & 5700 & 1.5 & 84 \\
\hline P1LC & 3700 & 5700 & 1.6 & 46 \\
\hline P2LC & 3400 & 5900 & 1.7 & 66 \\
\hline \multicolumn{5}{c}{${ }^{[\mathrm{a}]}$ Polystyrene standard. }
\end{tabular}

P1T: 2,5 -Bis-trimethylstannyl thiophene $(0.1 \mathrm{mmol})$ and mono-1 $(0.1 \mathrm{mmol})$ were dissolved in toluene. Tetrakis(triphenylphosphine)palladium $(0)\left(1.5 \times 10^{-3} \mathrm{~g}\right)\left[\mathrm{Pd}\left(\mathrm{PPh}_{3}\right)_{4}\right]$ was added after a reaction vessel was maintained at $97^{\circ} \mathrm{C}$. After $18 \mathrm{~h}$, the solution was dissolved in a minimal amount of chloroform and poured into a large amount of methanol, and stirred for $3 \mathrm{~h}$, then methanol was removed by decantation. The wash procedure in methanol was repeated. After collection of the polymer by decantation, the compound was dried under vacuum to yield the desired polymer P1T as a green solid (0.09 mmol, $\mathrm{Y}=90 \%)$.

P2T: P2T was prepared by the same procedure for the preparation of P1T. Quantities used; 5,5'-bis(trimethylstannyl)-2,2'-bithiophene $(0.1 \mathrm{mmol})$, mono $1(0.1 \mathrm{mmol})$, toluene $(0.5 \mathrm{~mL})$, and $\mathrm{Pd}\left(\mathrm{PPh}_{3}\right)_{4}\left(1.4 \times 10^{-3} \mathrm{~g}\right)$. P2T was obtained as a dark red solid $(0.084 \mathrm{mmol}, \mathrm{Y}=84 \%)$.

P1LC: $(R)$-LC $(0.4021 \mathrm{~g})$ was added to a reaction vessel and maintained at $97{ }^{\circ} \mathrm{C}$. At the temperature, the iridescent color due to selective reflection of cholesteric liquid crystal phase was confirmed. Then, in $(R)$-LC, 2,5-bis-trimethylstannyl thiophene (0.05 mmol), mono 1 (0.05 mmol), and $\mathrm{Pd}_{\left(\mathrm{PPh}_{3}\right)_{4}}$ $\left(1.4 \times 10^{-3} \mathrm{~g}\right)$ were dissolved, and stirred at $97{ }^{\circ} \mathrm{C}$. After $18 \mathrm{~h}$, the reaction mixture was dissolved in a minimal amount of chloroform and solution was poured into a large amount of acetone to remove the $(R)$-LC. After stirring the mixture in acetone for $3 \mathrm{~h}$, the acetone was removed by decantation. The wash procedure in acetone was repeated. Finally, the product was washed in methanol and dried in vacuum to yield P1LC as a yellow solid $(0.023 \mathrm{mmol}, \mathrm{Y}=46 \%)$ 
P2LC: P2LC was prepared by the same procedure used for the preparation of P1LC. Quantities used; 5,5'-bis(trimethylstannyl)-2,2'-bithiophene (0.05 mmol), mono $1(0.05 \mathrm{mmol}),(R)$-LC (0.4025 g), and $\mathrm{Pd}\left(\mathrm{PPh}_{3}\right)_{4}\left(1.1 \times 10^{-3} \mathrm{~g}\right) . \mathrm{P} 2 \mathrm{LC}$ was obtained as a red solid $(0.033 \mathrm{mmol}, \mathrm{Y}=66 \%)$.

Gel permeation chromatography (GPC) relative to polystyrene reference was carried out to estimate the molecular weight of the polymers. The number-average molecular weights $\left(M_{\mathrm{n}}\right)$ of P1T, P2T, P1LC, and P2LC ranged from 3,400 to 4,700. The weight-average molecular weights $\left(M_{\mathrm{w}}\right)$ of P1T, $\mathrm{P} 2 \mathrm{~T}, \mathrm{P} 1 \mathrm{LC}$, and P2LC ranged from 5,700 to 6,900. The synthesis yields of P1T and P2T were 90\% and $84 \%$, respectively, while the yields of P1LC and P2LC were somewhat low, namely $46 \%$ and $66 \%$, respectively. This may be due to the fact that the steric hindrance between the $(R)$-LC molecules and the monomers resulted in depression of the polymerization activity for P1LC and P2LC. The low molecular mass fractions of P1LC and P2LC were removed by purification resulting in the low synthesis yield.

\section{Results and Discussion}

\subsection{Infrared absorption spectra}

Figure 1 shows infrared (IR) absorption spectra of (R)-LC, P1LC, P2LC, P1T, and P2T. In the IR absorption spectra, P1LC and P1T exhibited the same features. Similarly, P2LC and P2T exhibited the same spectral shape. The GPC and IR absorption results suggested that the polymerization in the $(R)$-LC was successfully conducted.

Figure 1. Infrared (IR) absorption spectra for the (R)-LC, P1LC, P2LC, P1T, and P2T with the KBr method.

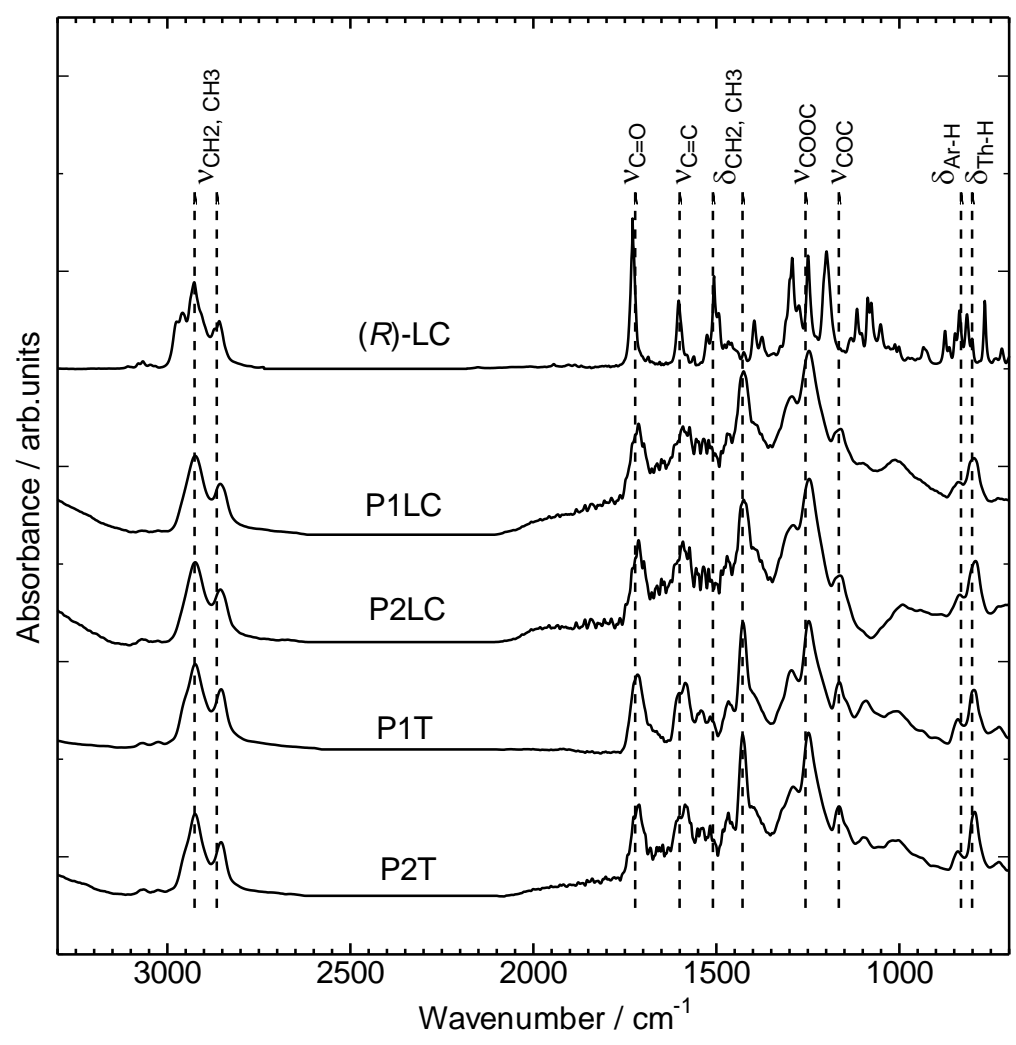




\subsection{Ultraviolet-visible absorption and photoluminescence spectra}

Figure 2 shows ultraviolet-visible (UV-vis) absorption spectra for the polymers in chloroform solutions. All polymers exhibited two absorption bands. The absorption bands of the polymers at short wavelengths were located at almost the same position $(284 \mathrm{~nm})$, which is characteristic of the $\pi-\pi^{*}$ transition of phenyl pyrimidine moiety in the side chains. On the other hand, the absorption bands at long wavelengths are attributable to the $\pi-\pi^{*}$ transition in the conjugated main chains. The maximum absorption wavelength $\left(\lambda_{\max }\right)$ at $389 \mathrm{~nm}$ for the P1LC is $5 \mathrm{~nm}$ longer than that for the P1T (384 $\mathrm{nm}$ ). Similarly, $\lambda_{\max }$ for P2LC at $425 \mathrm{~nm}$ is $13 \mathrm{~nm}$ longer than that for P2T (412 nm). These red shifts indicated a development of $\pi$-stacking between the polymers prepared in $(R)$-LC.

Figure 2. UV-vis absorption spectra for P1LC (gray solid line), P1T (gray dashed line), P2LC (black solid line), and P2T (black dashed line) in chloroform solutions $\left(c=5 \times 10^{-5} \mathrm{M}\right)$.

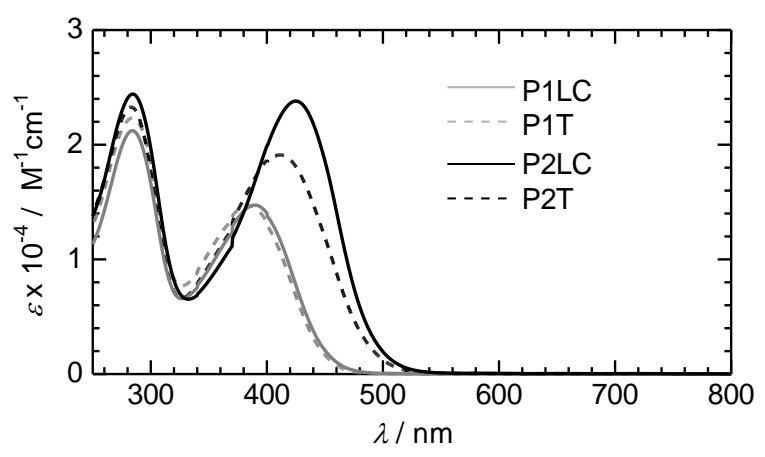

Here, an anisotropic propagation of the polymers may occur along the liquid crystalline order during the polymerization process in the $(R)$-LC medium. The thiophene units may stack in the narrow space between the liquid crystal molecules during the polymerization. The absorption bands of P2LC and P2T were located at longer wavelengths than those of P1LC and P1T because the introduction of a bithiophene unit into the main chain without the side chain provides an ample space between the substituents, and the space may improve coplanarity of the polymer. Besides, an increase in the ratio of the thiophene units leads to lower energy bandgap of the polymers, because in general, polythiophenes have lower energy bandgaps than the poly-p-phenylenes. Here, effective conjugation length of the present polymers may not be highly developed compared to non-substituted polyacetylene. Furthermore, molecular weights of the polymers are almost the same range. Therefore, in the present stage we do not consider dependence of molecular weight for the optical absorption results.

Figure 3 shows optical absorption spectra of P2T and P2LC at low concentration in chloroform solution (P2T, $C=9.6 \times 10^{-6} \mathrm{M}$; P2LC, $C=7.5 \times 10^{-6} \mathrm{M}$ ). The absorption maximum of P2LC displays $12 \mathrm{~nm}$ longer than that of P2T. Further, the absorption intensity is stronger than that of P2T. This may be due to the fact that an aggregation produced in the liquid crystal medium allows development of coplanarity between adjacent monomer repeat units in the main chain. 
Figure 3. UV-vis absorption spectra for P2T (yellow-green line) and P2LC (purple line) at low concentration in chloroform solutions.

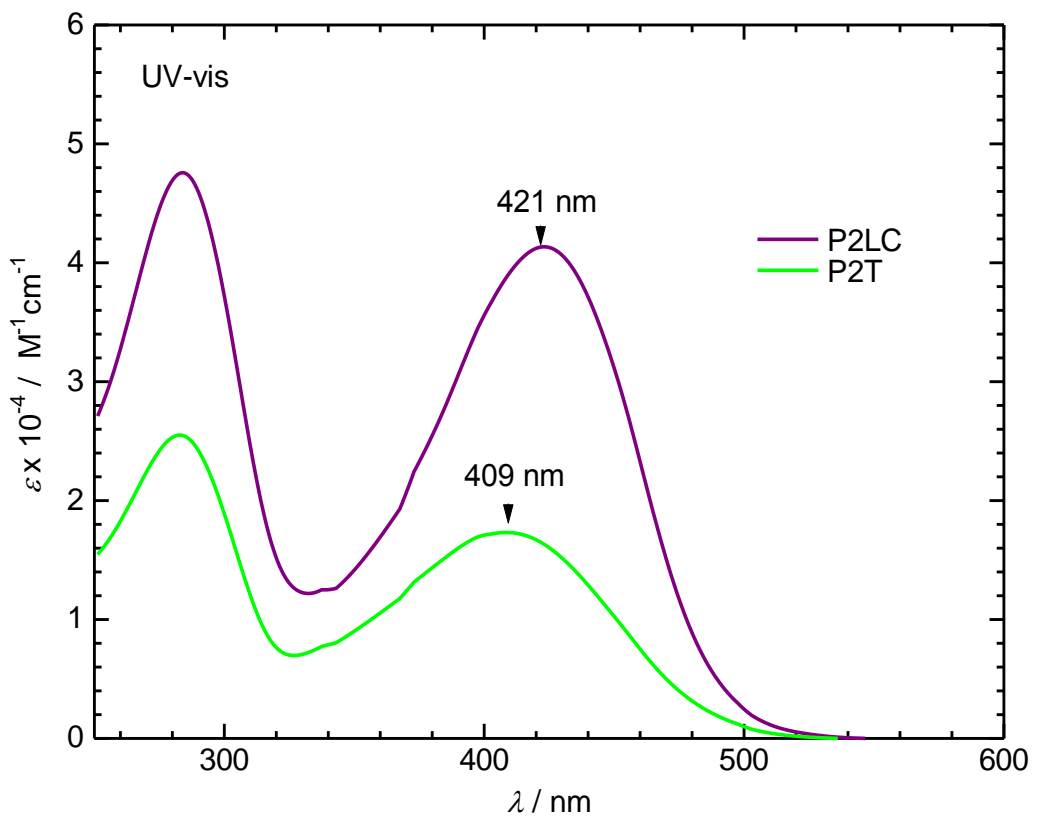

Figure 4 shows excitation spectra of P2T and P2LC for photoluminescence in chloroform solution. The normalized intensity of the polymers decreased with concentration. The intensities of P2LC are stronger than that of P2T at each concentration. The intensity maximum of the P2LC locates at long wavelengths compared to P2T. This may be due to the fact that restricted space of LC medium during the polymerization improves molecular packing between the main chains and affords good $\pi$-stacking to form. The $\pi$-stacking locks the aggregation for improvement of coplanarity. The red-shift observed for P2LC is due to development of electronic delocalization on the main chain. The result of excitation spectra is consistent with the absorption spectra of the polymers at low concentration.

Figure 4. Excitation spectra for P2T (left) and P2LC (right) in chloroform solutions.
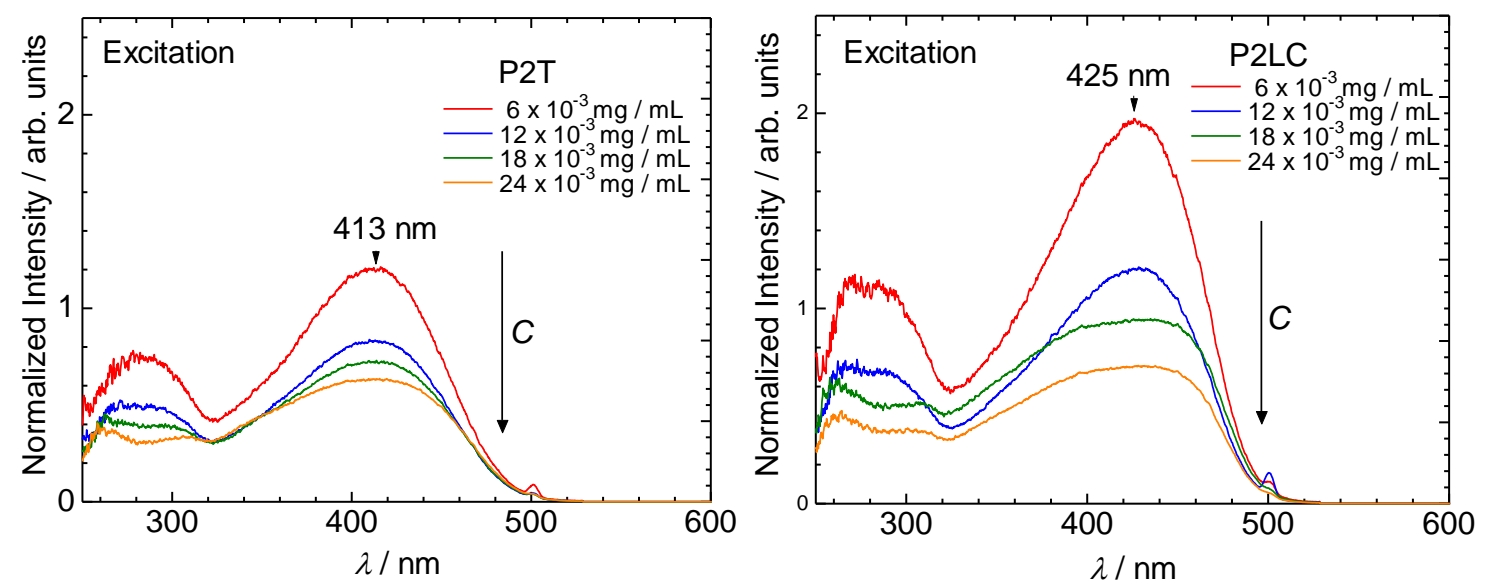

Figure 5 shows photoluminescence (PL) spectra for P2T and P2LC at low concentration in chloroform solution. The PL intensity of P2LC is stronger than that of P2T. Also, the PL maximum value of P2LC is located at slightly longer wavelengths $(2 \mathrm{~nm})$ compared to P2T. This result suggests 
that the aggregation produced by the liquid crystal medium during the polymerization reaction allows improvement of emission of the polymer.

Figure 5. Photoluminescence (PL) spectra for P2T (left) and P2LC (right) in chloroform solutions.
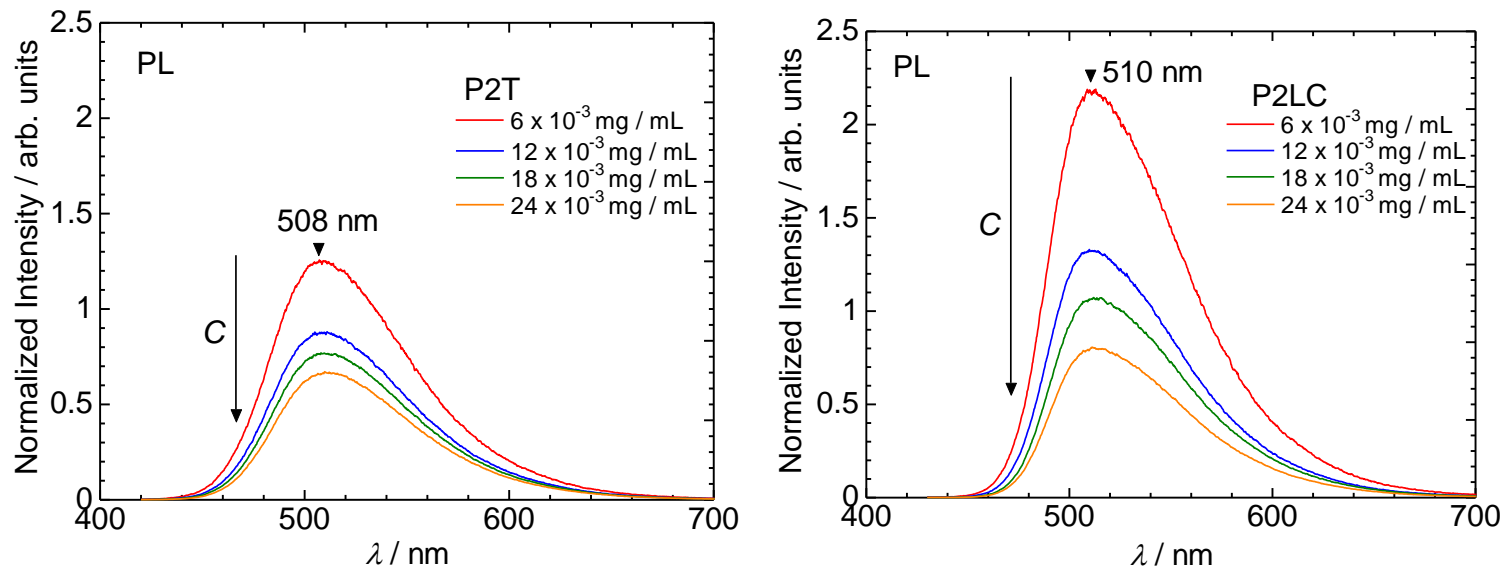

\subsection{Polarizing optical microscopy observation of polymerization media}

Figures 6 and 7 show polarizing optical microscopic (POM) images of the $(R)$-LC media containing the monomers (before polymerization), the resultant polymers and catalysts (after polymerization) at $97{ }^{\circ} \mathrm{C}$. The Grandjean texture (planer texture) due to the cholesteric liquid crystallinity was observed in all reaction mixtures. Although the Grandjean texture in Figure 7(b) is small, the sample exactly shows cholesteric phase with birefringence. The visual inspection confirmed that the LC reaction mixture maintained the LC phase throughout the polymerization reaction. These results indicated that $(R)$-LC in the LC state can function as the polymerization medium.

Figure 6. Polarizing optical microscopic (POM) images of the reaction mixture of P1LC before polymerization (a) and P1LC after polymerization (b).

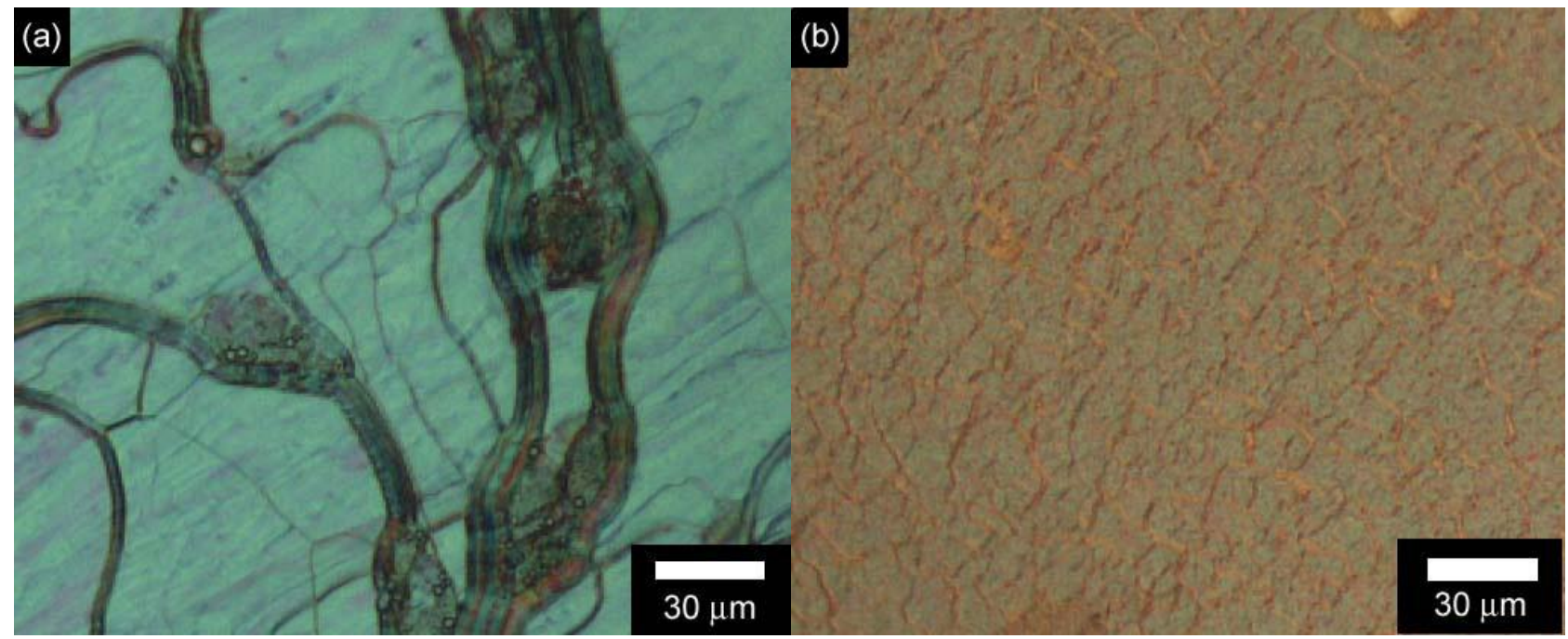


Figure 7. POM images of the reaction mixture of P2LC before polymerization (a) and P2LC after polymerization (b).

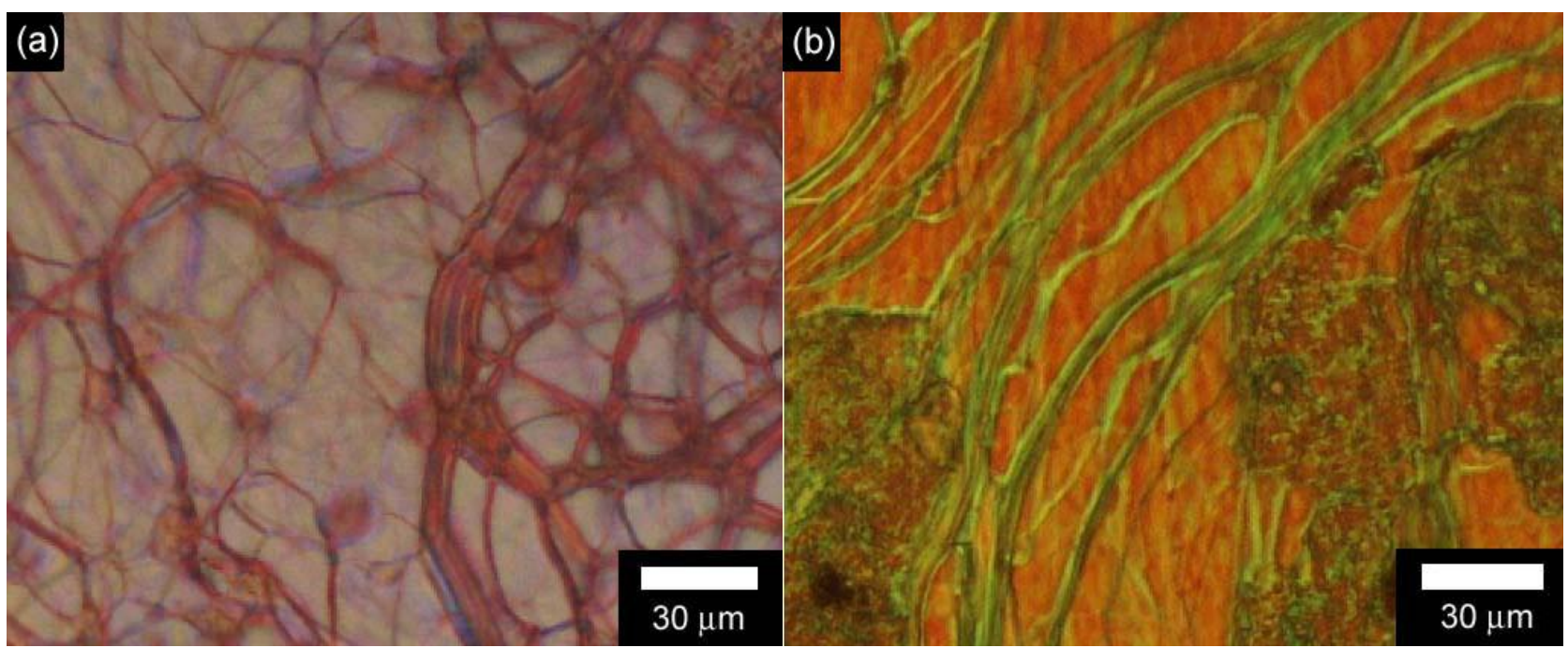

The color of the texture derived from optical retardation, and it depends on each condition of POM observations (i.e., sample thickness in the cell or substrate state). The optical textures demonstrate that the helical axis of all of the samples locates normal direction to the substrate (this is a characteristic of Grandjean texture).

\subsection{Phase transition}

Phase transition temperatures for the polymers thus prepared were determined with both differential scanning calorimetry (DSC) (Figure 8) and POM observations. The DSC signals did not display high resolution because the enthalpy change involves both phase transition of the side chain and the main chain. Furthermore, the dispersion of the molecular weight of the main chain in the polymer complicates the transition.

All polymers exhibited blushes under the POM, which is typical of Schlieren textures of nematic LC (NLC) order. Both polymers prepared in isotropic solution (toluene) and polymers prepared in LC show nematic phase. The polymer aggregation is released with temperature during the heating process, and the polymers form liquid crystal order. Continuously, the phase transition from LC (mesophase) to isotropic state (liquid phase) occurs in the heating process. Note that the mono- 1 shows no LC. On the other hand, the corresponding polymer exhibits thermotropic liquid crystallinity. This phenomenon can be referred to as the polymer effect, because the polymerization produces liquid crystallinity in this system. Sequential arrangement of the side chain attached onto the main chain and the rigid form of the main chain of polymers allow formation of polymer LC.

The transition temperatures from isotropic phase to LC phase of P1T, P2T, and P1LC were in the range of $135-160{ }^{\circ} \mathrm{C}$, while the transition temperature from isotropic phase to LC phase of P2LC was $220{ }^{\circ} \mathrm{C}$. This high phase transition temperature of P2LC suggested that P2LC may have a higher molecular weight than that of the other polymers. Alternatively, P2LC may have a tight aggregated structure. The GPC measurement of P2LC estimated a lower molecular weight fraction because the polymer solution was filtered using a microporous membrane to exclude the insoluble 
fraction. This procedure is required for GPC measurements. The optical textures of P1LC (at $120{ }^{\circ} \mathrm{C}$ ), P2LC (at $200{ }^{\circ} \mathrm{C}$ ), P1T (at $130{ }^{\circ} \mathrm{C}$ ), and P2T (at $120^{\circ} \mathrm{C}$ ) were observed with the POM (Figures 9 and 10).

Liquid crystallinity of polymers can be derived from the orientation of the main chain. Here, the main chain plays a role of a mesogen with the cooperation of side chain LC group. This may be referred to as super mesogen form. This is because both the main chain and the side chain did not independently exhibit liquid crystallinity. The super mesogen form was observed for the main chain-side chain type polymers in the previous study [18]. XRD measurements can further confirm the structure of the polymer, and may display broad signal (hallow) such as typical nematic phase.

Figure 8. DSC curves of P1T (a), P2T (b), P1LC (c), and P2LC (d).
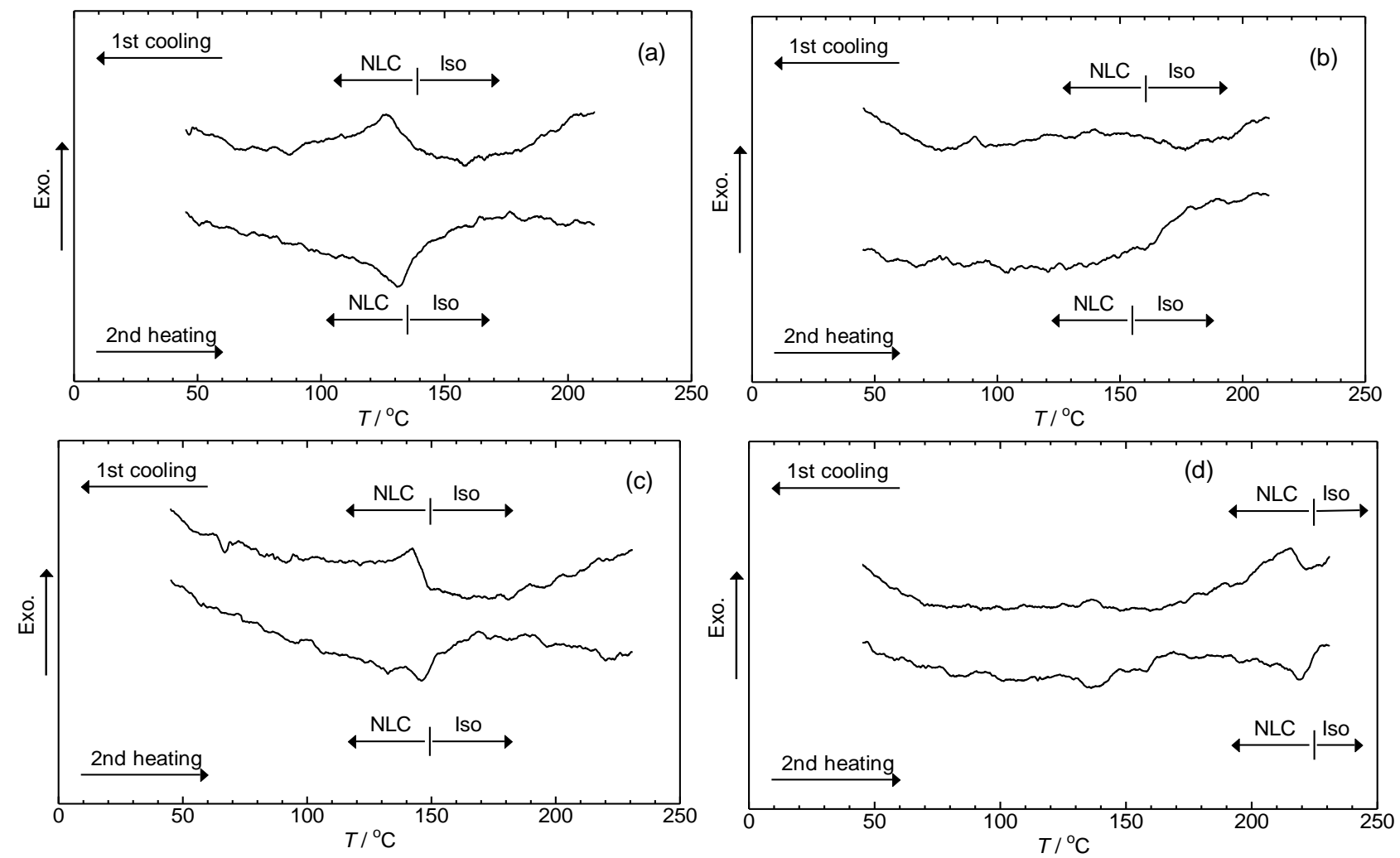

Figure 9. POM images of $\mathrm{P} 1 \mathrm{~T}$ at $130{ }^{\circ} \mathrm{C}$ (a) and $\mathrm{P} 2 \mathrm{~T}$ at $120^{\circ} \mathrm{C}(\mathbf{b})$.

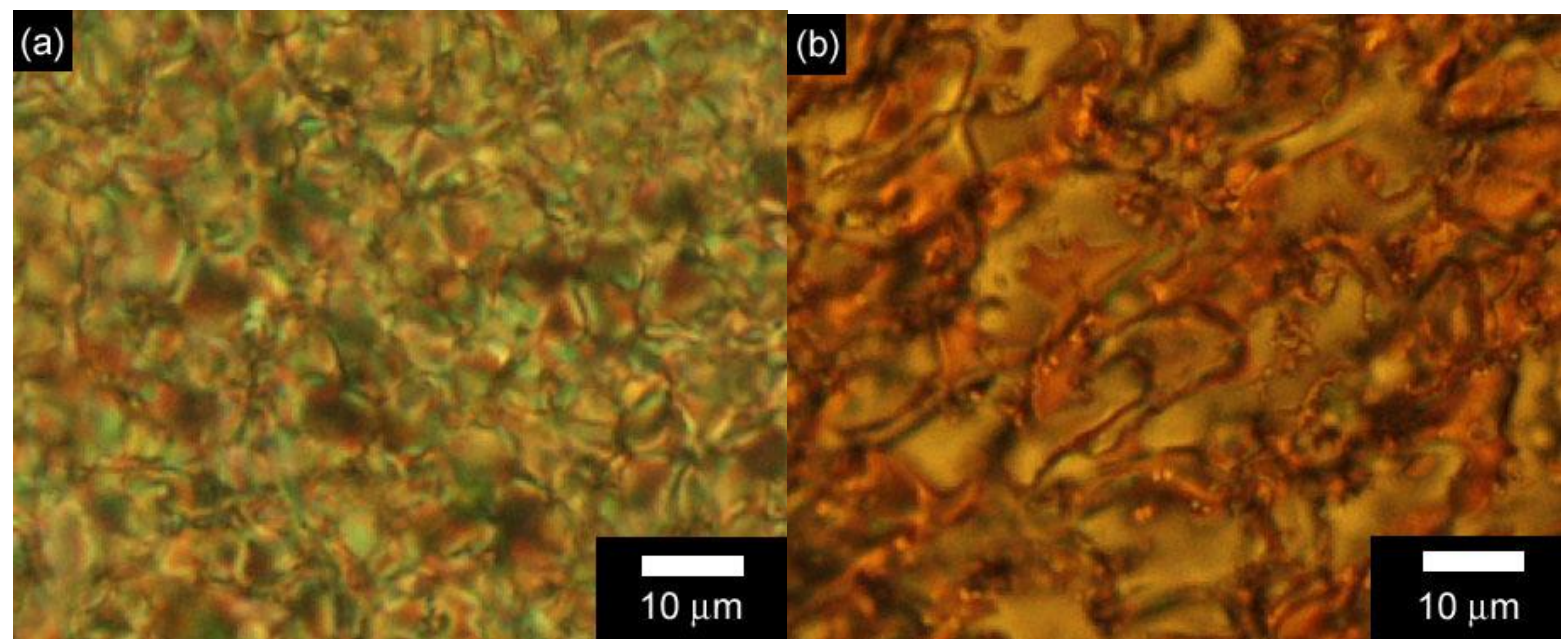


Figure 10. POM images of P1LC at $120^{\circ} \mathrm{C}$ (a) and $\mathrm{P} 2 \mathrm{LC}$ at $200{ }^{\circ} \mathrm{C}(\mathbf{b})$.

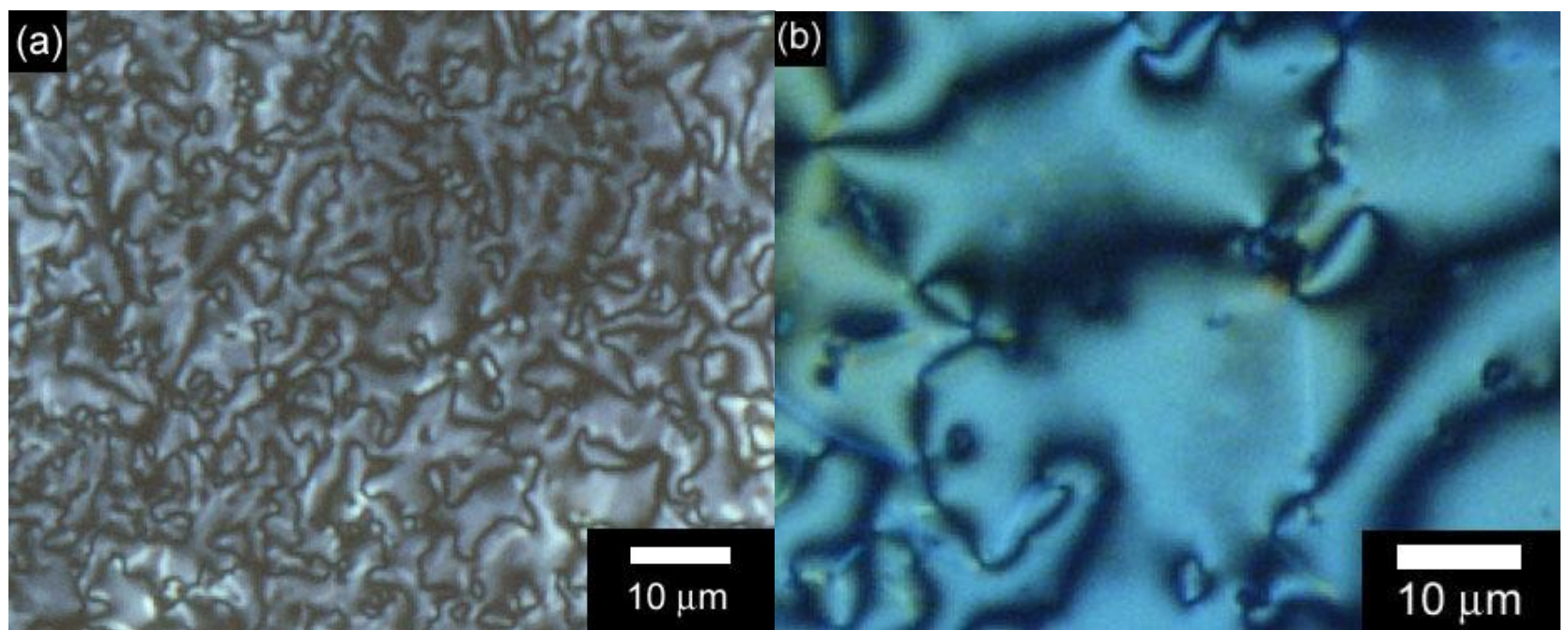

The plausible structure is shown in Figure 11. The texture of the polymer (Schlieren, nematic) is different from that of the $(R)$-LC medium (Grandjean, cholesteric). This result supports the fact that the polymer contains no LC medium.

Figure 11. A plausible structure of the main chain and side chain cooperation LC system (super mesogen).

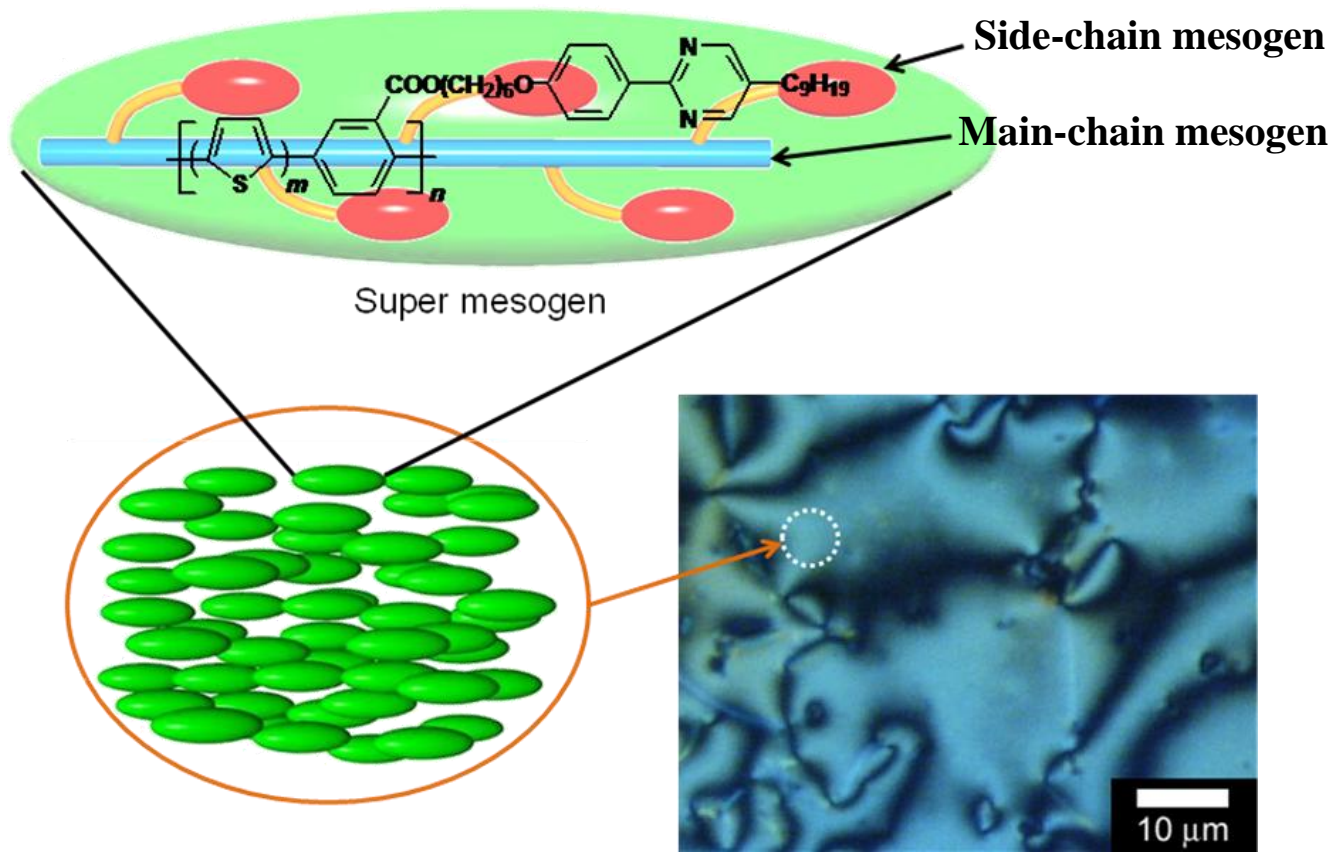

If the sample contains some cholesteric molecules, the polymer would show cholesteric phase because a small amount of chiral compounds induces cholesteric phase in the entire system. A set of polymerization results in this study demonstrated that the reaction in the liquid crystal medium successfully produced polymers with bulky pyrimidine LC group. 


\section{Conclusions}

We successfully carried out the polycondensation of aromatic monomers with the bulky pyrimidine substituent in a LC as the polymerization solvent. The resultant polymers show nematic LCs accompanied by the formation of super mesogens. The polymers thus prepared in $(R)$-LC have relatively long effective $\pi$-conjugation length due to development of coplanarity derived from $\pi$-stacking. The results of this study demonstrated the possibility of conducting various polymerization reactions in ordered solvents.

\section{Acknowledgements}

We would like to thank for Midori Chemical Co., Ltd. for providing the pyrimidine LC precursor compound. The gratitude is extended to the Chemical Analysis Center of University of Tsukuba and the glass work shop of University of Tsukuba.

\section{References}

1. Baxter, B.C.; Gin, D.L. Synthesis and Polymerization of a Chiral Liquid Crystal Diacrylate Exhibiting Smectic A* and C* Phases. Macromolecules 1998, 31, 4419-4425.

2. Okoshi, K.; Watanabe, J. Alternating Thick and Thin Layers Observed in the Smectic Phase of Binary Mixtures of Rigid-Rod Helical Polysilanes with Different Molecular Lengths. Macromolecules 2010, 43, 5177-5179.

3. Chen, S.H.; Mastrangelo, J.C.; Conger, B.M.; Kende, A.S.; Marshall, K.L. Synthesis and Characterization of Thermotropic Chiral-Nematic Polythiophenes. Macromolecules 1998, 31, 3391-3393.

4. Longa, L.; Pająk, G.; Wydro, T. Stability of Biaxial Nematic Phase for Systems with Variable Molecular Shape Anisotropy. Phys. Rev. E 2007, 76, 011703.

5. Wang, J.W.; Meng, F.B.; Li, Y.H.; Zhang, B.Y. Synthesis and Characterization of Side-Chain Cholesteric Liquid-Crystalline Polysiloxanes Containing Different Space Groups. J. Appl. Polym. Sci. 2009, 111, 2078-2084.

6. Yoshida, H.; Inoue, Y.; Isomura, T.; Matsuhisa, T.; Fujii, A.; Ozaki, M. Position Sensitive, Continuous Wavelength Tunable Laser Based on Photopolymerizable Cholesteric Liquid Crystals with an In-Plane Helix Alignment. Appl. Phys. Lett. 2009, 94, 093306.

7. Bobrovsky, A.; Boiko, N.; Shibaev, V.P.; Zavarzin, I.; Kalik, M.; Krayushkin, M. Cholesteric Mixture Containing Chiral-photochromic and Diarylethene Dopants as Novel Material with Dual Photochromism. Polym. Adv. Technol. 2002, 13, 595-600.

8. Liedtke, A.; Lei, C.; O’Neill, M.; Dyer, P.E.; Kitney, S.P.; Kelly, S.M. One-Step Photoembossing for Submicrometer Surface Relief Structures in Liquid Crystal Semiconductors. ACS. Nano. 2010, 4, 3248-3253.

9. Ren, W.; McMullan, P.J.; Griffin, A.C. Stress-strain Behavior in Main Chain Liquid Crystalline Elastomers: Effect of Crosslinking Density and Transverse Rod Incorporation on "Poisson's Ratio”. Phys. Status Solid B 2009, 246, 2124-2130. 
10. Zhu, Z.; Swager, T.M. Conjugated Polymer Liquid Crystal Solutions: Control of Conformation and Alignment. J. Am. Chem. Soc. 2002, 124, 9670-9671.

11. Nishizawa, K.; Nagano, S.; Seki, T. Novel Liquid Crystalline Organic-Inorganic Hybrid for Highly Sensitive Photoinscriptions. Chem. Mater. 2009, 21, 2624-2631.

12. Kim, N.; Choi, J.; Chien, L.C.; Kyu, T. Phase Equilibria of a Mixture of Side-Chain Liquid Crystalline Polymer and Low Molecular Mass Liquid Crystal. Macromolecules 2007, 40, 9582-9589.

13. Goto, H. Cholesteric Liquid Crystal Inductive Asymmetric Polymerization: Synthesis of Chiral Polythiophene Derivatives from Achiral Monomers in a Cholesteric Liquid Crystal. Macromolecules 2007, 40, 1377-1385.

14. Kawabata, K.; Goto, H. Periodic Structure in a Fluorene-based Polymer Prepared by Electrochemical Polymerization. Chem. Lett. 2009, 38, 706-707.

15. Araya, K.; Mukoh, A.; Narahara, T.; Shirakawa, H. Polymerization of Acetylene in Liquid Crystal Solvent. Chem. Lett. 1984, 13, 1141-1142.

16. Kilbinger, A. F. M.; Feast, W. J. Solution Processable Alternating Oligothiophene-PEO-blockcopolymers: Synthesis and Evidence for Solvent Dependent Aggregation. J. Mater. Chem. 2000, 10, 1777-1784.

17. Mitsunobu, O.; Yamada, M. Preparation of Esters of Carboxylic and Phosphoric Acid via Quaternary Phosphonium Salts. Bull. Chem. Soc. Jpn. 1967, 40, 2380-2382.

18. Kawabata, K.; Goto, H. Liquid Crystalline $\pi$-Conjugated Copolymers Bearing a Pyrimidine Type Mesogenic Group. Materials 2009, 2, 22-37.

(C) 2010 by the authors; licensee MDPI, Basel, Switzerland. This article is an open access article distributed under the terms and conditions of the Creative Commons Attribution license (http://creativecommons.org/licenses/by/3.0/). 